

\title{
V. GRUPO TRANSFRONTERIZO/CROSS-FRONTIER GROUP, MEMORÁNDUM DE ENTENDIMIENTO CON EL GOBIERNO DE GIBRALTAR (2017) Y REGLAMENTO DE FUNCIONAMIENTO (2018)
}

\author{
Inmaculada GONZÁLEZ GARCÍA*; Alejandro DEL VALLE GÁLVEZ; \\ Lorena CALVO MARISCAL; Juan Domingo TORREJÓN RODRÍGUEZ
}

\section{Documentación incluida en este apartado:}

DOCUMENTO 1. Memorándum de Entendimiento para fortalecer la cooperación entre el Gobierno de Gibraltar, el Grupo Transfronterizo de Gibraltar y el Campo de Gibraltar, 7 de junio de 2017. Memorandum of Understanding to strengthen cooperation between HM Government of Gibraltar and the Cross-Frontier Group for Gibraltar and the Campo de Gibraltar, 7 june 2017.

DOCUMENTO 2. Nuevo Reglamento de Funcionamiento del Grupo Transfronterizo de Gibraltar y el Campo de Gibraltar, 26 de enero de 2018**.

\footnotetext{
* Esta Sección se enmarca en el Proyecto de Investigación financiado por la Fundación Pública Andaluza Centro de Estudios Andaluces (PRY205/19): “La incidencia del 'Brexit' en la cooperación transfronteriza entre Gibraltar-Campo de Gibraltar y Andalucía”, coordinado por la Profesora Inmaculada González García. Período de vigencia, 2020-2022.

** Documento facilitado por D. Juan Carmona de Cozar, del Grupo Transfronterizo, a quien agradecemos su colaboración con la Revista Cuadernos de Gibraltar/Gibraltar Reports, y los Proyectos de Investigación del Area de Derecho Internacional y Derecho Europeo de Cádiz.
} 


\section{COMUNICADO}

\section{Gobierno de Gibraltar}

\section{El Gobierno de Gibraltar firma un memorándum de entendimiento con el Grupo Transfronterizo}

Gibraltar, 7 de junio de 2017

El Gobierno del Peñón ha firmado hoy un memorándum de entendimiento con el Grupo Transfronterizo de Gibraltar y el Campo de Gibraltar. El objetivo común expuesto en el memorándum es el de facilitar y promover el crecimiento de las economías de Gibraltar y del Campo de Gibraltar y garantizar una frontera fluida.

El Ministro Principal, Fabián Picardo, ha firmado en nombre del Gobierno de Gibraltar, y por el Grupo Transfronterizo lo han hecho representantes de las asociaciones y organizaciones que lo integran: Manuel Triano (Comisiones Obreras), Ángel Serrano (UGT), Louis González (Unite the Union), Stuart Borastero (sindicato gibraltareño GTA/NASUWT), Wendy Cumming (sindicato gibraltareño GGCA), John Ísola (Cámara de Comercio de Gibraltar), Kamlesh Khubchand (Federación de la Pequeña Empresa de Gibraltar), y Lorenzo Pérez-Periáñez (APYMELL de La Línea). La firma ha tenido lugar en el № 6 de Convent Place.

\section{Nota a redactores:}

Esta es una traducción realizada por la Oficina de Información de Gibraltar. Algunas palabras no se encuentran en el documento original y se han añadido para mejorar el sentido de la traducción. El texto válido es el original en inglés.

Para cualquier ampliación de esta información, rogamos contacte con Oficina de Información de Gibraltar

Miguel Vermehren, Madrid, miguel@infogibraltar.com, Tel 609004166

Sandra Balvín, Campo de Gibraltar, sandra@infogibraltar.com, Tel 637617757

Eva Reyes Borrego, Campo de Gibraltar, eva@infogibraltar.com, Tel 619778498

Web: www.infogibraltar.com, web en inglés: $\underline{w w w . g i b r a l t a r . g o v . g i / p r e s s-o f f i c e ~}$ Twitter: @InfoGibraltar 

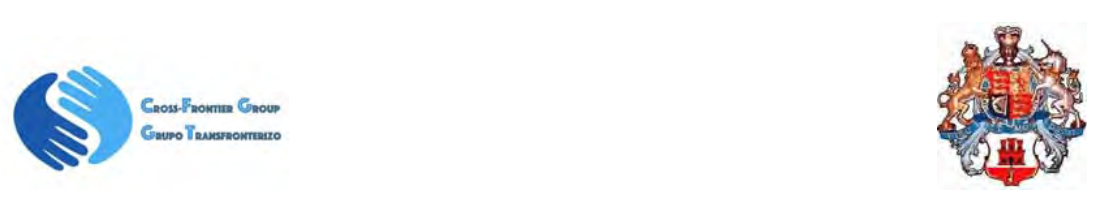

\section{MEMORANDUM DE ENTENDIMIENTO PARA FORTALECER LA COOPERACION ENTRE EL GOBIERNO DE GIBRALTAR, EL GRUPO TRANSFRONTERIZO DE GIBRALTAR Y EL CAMPO DE GIBRALTAR.}

(1) Este memorándum de entendimiento se formaliza el 7 de junio de 2017 entre el gobierno de Gibraltar y el Grupo Transfronterizo con el objetivo común de facilitar y promover el crecimiento de las economías del Campo de Gibraltar y de Gibraltar. El Gobierno de Gibraltar y el Grupo Transfronterizo, mediante el presente memorándum, establecen los beneficios que ofrece Gibraltar para el Campo de Gibraltar y viceversa. El efecto económico positivo entre ambas zonas podría ser todavía mayor y generar aún más riqueza.

(2) El objetivo del presente memorándum es establecer un compromiso de cooperación y colaboración, asegurar un funcionamiento fluido de la frontera, desarrollar el potencial económico y mejorar las oportunidades de empleo a ambos lados de la frontera.

(3) Ambos signatarios se comprometen a trabajar conjuntamente y compartir aquellos recursos que beneficien a sus respectivas organizaciones y al público en general a ambos lados de la frontera, siempre que no haya conflictos de interés.

(4) MODALIDADES DE COOPERACION

Las partes podrán fomentar la colaboración establecida en la introducción del memorándum a través de las siguientes áreas de cooperación.

- Intercambio de información.

- Desarrollo de proyectos conjuntos y actividades relacionadas.

- Intercambio de especialistas, recursos humanos y materiales.

- Organización de talleres, conferencias y seminarios que abarquen las áreas de cooperación.

- Uso de instalaciones y recursos educativos para actividades de formación.

- Mantener y mejorar la circulación de personas a través de la frontera.

- Mantener y mejorar el tránsito de vehículos y mercancías a través de la frontera y establecer un marco jurídico para tal fin.

- Mantener el empleo actual e impulsar la creación de empleo.

- Estimular la demanda de bienes y servicios en los distintos sectores empresariales

- Ofrecer facilidades, información y asistencia a las empresas locales de Gibraltar y el Campo de Gibraltar para que puedan establecer a ambos lados de la frontera.

- Impulsar la creación de una zona fiscal en la Línea de la Concepción que permita que las empresas gibraltareñas puedan establecer ahí su actividad, como una fuente de creación de empleo para el Campo de Gibraltar y Gibraltar.

- Estudiar estrategias alternativas de desarrollo empresarial y cooperación a ambos lados de la frontera. 
V. Grupo Transfronterizo/Cross-Frontier Group, Memorándum de Entendimiento con el Gobierno de Gibraltar (2017) y

Reglamento de funcionamiento (2018)
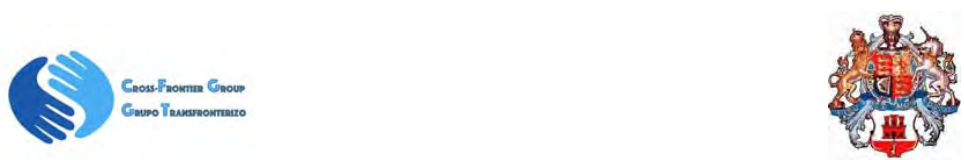

(5) Un subcomité, cuya composición se acordará por ambas partes, se encargará de coordinar las actividades específicas entre los signatarios de este memorándum de entendimiento, por el cual los miembros de este subcomité podrán recibir apoyo de agencias externas que no estén adscritas a este ME.

(6) Todos los signatarios están obligados a tratar cualquier asunto discutido con la mayor confidencialidad, por lo que sólo se informará a los medios de aquellos acuerdos o áreas de cooperación sobre los cuales ya se haya alcanzado un pacto.

(7) El presente memorándum de entendimiento entrará en vigor a partir de la fecha de su firma y tendrá una vigencia de tres años, prorrogable automáticamente por periodos iguales, a menos que cualquiera de las partes manifieste su decisión de darlo por terminado, en cuyo caso deberá informar de su decisión a la otra por escrito.

(8) El presente memorándum podrá ser modificado si así lo conciertan las partes y lo consignan por escrito..

(9) Este memorándum no se verá afectado por acuerdos independientes celebrados entre cualquiera de los signatarios, ya sean los miembros del Grupo Transfronterizo o el Gobierno de Gibraltar, o por cualquier afiliación política individual o colectiva.

(10) La resolución anticipada del presente acuerdo no afectará a la conclusión de las actividades de cooperación que hayan sido formalizadas durante su vigencia.

Firmado en Gibraltar el día 7 de junio de 2017 
HM GOVERNMENT OF GIBRALTAR:

CHIEF MINISTER

POR EL GRUPO TRANSFRONTERIZO: 
V. Grupo Transfronterizo/Cross-Frontier Group, Memorándum de Entendimiento con el Gobierno de Gibraltar (2017) y Reglamento de funcionamiento (2018)

\section{PRESS RELEASE}

No: $371 / 2017$

Date:7th June 2017

\section{HMGOG Signs Memorandum Of Understanding With Cross Frontier Group}

HM Government of Gibraltar today signed a Memorandum of Understanding with the Cross Frontier Group for Gibraltar and the Campo Area. The common aim of the Memorandum is to facilitate and promote growth in the economies of Gibraltar and the Campo and ensure a fluid frontier.

The Chief Minister, the Hon Fabian Picardo, QC MP, signed on behalf of HM GOG and representatives of the different organisations in the Cross Frontier Group signed on its behalf. The signing took place at No. 6 Convent Place. 


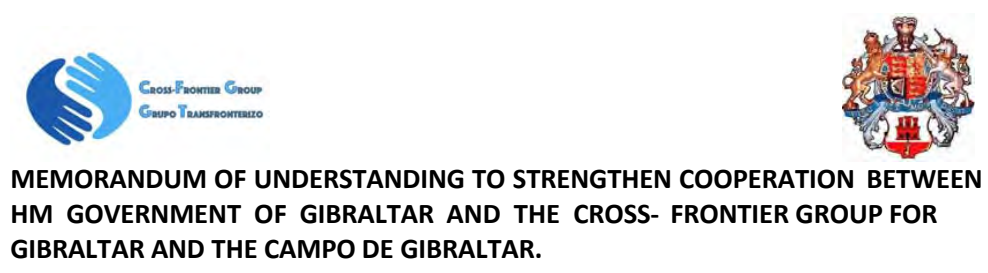

(1) This memorandum of understanding is entered into on the $7^{\text {th }}$ day of June 2017 between Her Majesty's Government of Gibraltar and the Cross-Frontier Group with the common aim of facilitating and promoting growth in the economies of Gibraltar and the Campo de Gibraltar. Through this Memorandum, Her Majesty's Government of Gibraltar and the Cross-Frontier Group set out the advantages that Gibraltar offers the Campo de Gibraltar and vice versa. The positive economic impact on both areas could be greater and generate much more wealth.

(2) The aim of this document is to establish a commitment to cooperation, and collaboration, to ensure a fluid border, develop economic potential and improve employment opportunities on both sides of the border.

(3) Both signatories undertake to work together and share resources of benefit to their respective organisations and the general public on both sides of the border and providing that there is no conflict of interest.

(4) FORMS OF COOPERATION

Both parties will be able to promote collaboration as set out in the introduction of this Memorandum through the following areas of cooperation.

— Information Exchange.

- Development of joint projects and activities in support thereof.

- Exchange of experts, human resources and materials

- Organization of workshops, conferences and seminars covering the areas of cooperation.

— Use of facilities and educational resources for training.

- Maintaining and improving the movement of people across the border.

- Maintaining and improving the movement of vehicles and goods across the border and creating a legal framework to achieve this.

- Maintaining and creating new employment.

- Stimulating demand for goods and services in the various business sectors.

- Offering facilities, information and assistance to local businesses in Gibraltar and the Campo the Gibraltar to establish themselves on either side of the border.

- Promote the creation of a tax zone in La Línea de la Concepción allowing Gibraltar based companies the opportunity to set up operations, as a source of job creation for the entire Campo de Gibraltar and Gibraltar. 
V. Grupo Transfronterizo/Cross-Frontier Group, Memorándum de Entendimiento con el Gobierno de Gibraltar (2017) y

Reglamento de funcionamiento (2018)

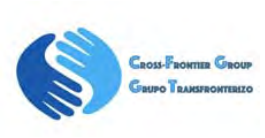

- Research other areas of business development and cooperation on both sides of the border.

(5) A subcommittee, the composition of which will be agreed by both parties, will be responsible for coordinating specific activities between the signatories of this Memorandum and whereby the members of this subcommittee could receive the support of external Agencies that do not form part of this MOU.

(6) All signatories are obliged to treat all matters discussed with the utmost confidentiality and the media shall only be informed of any agreements or areas of cooperation once this has been agreed by all the signatories.

(7) This Memorandum will come into effect on the day that it is signed and will be valid for a period of three years. It will be automatically renewed for equal periods of time, unless either party expresses otherwise, in which case' the party terminating the Agreement would be required to write directly to the other party informing it of its decision.

(8) This Memorandum may be amended by mutual agreement of the parties in writing stating the effective date of the amendments.

(9) This Memorandum is free of any other separate agreements entered into by either party, be that between the members of the Cross-Frontier Group or by HM Government of Gibraltar. It is also free of any political affiliations be they independent or collective.

(10)The early termination of this Agreement will not affect any separate activities that may have been agreed to, formally, during the course of the Agreement.

Signed in Gibraltar this $7^{\text {th }}$ Day of June, 2017 
HM GOVERNMENT OF GIBRALTAR:

CHIEF MINISTER

GRUPO TRANSFRONTERIZO: 


\title{
DOCUMENTACIÓN
}

V. Grupo Transfronterizo/Cross-Frontier Group, Memorándum de Entendimiento con el Gobierno de Gibraltar (2017) y Reglamento de funcionamiento (2018)

\author{
GRUPO TRANSFRONTERIZO
}

26 de enero de 2018

REGLAMENTO DE FUNCIONAMIENTO GRUPO TRANSFRONTERIZO 


\section{GRUPO TRANSFRONTERIZO}

El Grupo Transfronterizo es un organismo de coordinación integrado por organizaciones sindicales y empresariales de Gibraltar y del Campo de Gibraltar.

\section{COMPOSICIÓN}

Gibraltar:

- CHAMBER OF COMMERCE

- GGCA

- GFSB

- GTA / NASUWT

- UNITE THE UNION

\section{Campo de Gibraltar}

- APYMELL

- COMISIONES OBRERAS

- UGT

- confederación de EMPREsarios de cádiz

- cÁmara de comercio del campo de gibraltar

\section{Marco Socio-Demográfico}

El ámbito territorial de acción de los integrantes del grupo comprende el territorio del Campo de Gibraltar y Gibraltar. Del contexto social en el que se enclava podemos destacar:

REGLAMENTO DE FUNCIONAMIENTO GRUPO TRANSFRONTERIZO 
V. Grupo Transfronterizo/Cross-Frontier Group, Memorándum de Entendimiento con el Gobierno de Gibraltar (2017) y

Reglamento de funcionamiento (2018)

\section{DOC}

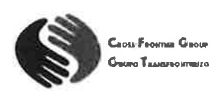

\section{La Línea de la Concepción v el Campo de Gibraltar (Cádiz)}

La Línea tiene una población de aproximadamente 69.000 personas con 20.500 trabajadores registrados y una tasa de desempleo del $37 \%$ (fuente: INEM). Toda la población del Campo de Gibraltar (hinterland español) es de unas 264.000 personas.

Una vez que la frontera estuvo totalmente abierta por España en1985, la economía de La Línea empezó a crecer más como consecuencia del desarrollo económico de Gibraltar. Gibraltar es una economía basada en el sector terciario (servicios). Por lo tanto, La Línea y las ciudades vecinas de la región del Campo históricamente han aportado muchas de las materias primas básicas que necesita Gibraltar para el propio desarrollo: materiales de construcción, muebles, repuestos, maquinaria, equipo de catering, alimentos y así sucesivamente.

Además, La Línea y las ciudades vecinas en el Campo de Gibraltar se han beneficiado de otras maneras. Los Gibraltareños y los trabajadores transfronterizos que van a Gibraltar, pero viven en España han invertido cantidades cada vez mayores de sus sueldos en España en los últimos años: en consumo, restaurantes, bares, hoteles, tiendas y grandes almacenes. Todo ello ha impulsado las propias economías locales y se ha creado empleo.

La Línea cuenta con muy poca industria, y hasta ahora, con muy poco apoyo de los distintos gobiernos, la ciudad ha llegado a depender de sus lazos comerciales con Gibraltar.

Como parte de acuerdos políticos anteriores, los tres gobiernos implicados acordaron construir una pieza contigua a la nueva terminal aérea de Gibraltar en la frontera, para pasar a La Línea, en un esfuerzo por desarrollar un aeropuerto regional común. Aunque en Gibraltar la nueva terminal aérea está completada y en pleno funcionamiento, a la fecha, en La Línea el enlace aún no

REGLAMENTO DE FUNCIONAMIENTO GRUPO TRANSFRONTERIZO 
ha comenzado. Este es un ejemplo de la falta de inversiones públicas. Del mismo modo, La Línea cuenta con un puerto, que fue comenzado en 1980 , pero nunca se ha optimizado su desarrollo de forma que sirva como terminal comercial, a pesar de su potencial, actualmente dentro del Puerto Bahía de Algeciras. Estas dos oportunidades de la ciudad podrían ayudar a generar mucho empleo y crecimiento económico. Por diferentes motivos, en los dos sistemas de más potencialidad de la ciudad, su progreso se ha detenido.

En un estudio llevado a cabo en el otoño de 2013 por la Asociación de la Pequeña y Mediana Empresa de La Línea (APYMELL), entre sus miembros se hace la pregunta de en qué medida sus empresas dependen de Gibraltar y también se les pidió estimar la pérdida de negocio sufrido desde que los controles y las colas comenzaron en agosto 2013 .

\begin{tabular}{|l|c|c|c|}
\hline Sector & $\begin{array}{c}\text { Empresas } \\
\text { encuestadas }\end{array}$ & $\begin{array}{c}\text { \% de clientela } \\
\text { desde Gibraltar }\end{array}$ & $\begin{array}{c}\text { Descenso } \\
\text { estimado en } \\
\text { ventas desde } \\
\text { Agosto 2013 }\end{array}$ \\
\hline Comercio & 144 & $30 \%$ & $28 \%$ \\
\hline $\begin{array}{l}\text { Hostelería (bares, } \\
\text { cafes, restaurantes } \\
\text { etc) }\end{array}$ & 44 & $33 \%$ & $32 \%$ \\
\hline \begin{tabular}{l} 
General Retail \\
\hline $\begin{array}{l}\text { Servicios } \\
\text { profesionales }\end{array}$
\end{tabular} & 16 & $24 \%$ & $26 \%$ \\
\hline
\end{tabular}


V. Grupo Transfronterizo/Cross-Frontier Group, Memorándum de Entendimiento con el Gobierno de Gibraltar (2017) y

Reglamento de funcionamiento (2018)

\section{DOC}

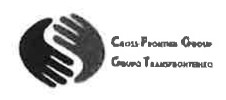

\section{Cámara de Comercio de Gibraltar Estudio de Impacto Económico:}

Este estudio se encargó en un intento por conocer la importancia del comercio transfronterizo entre Gibraltar y el Campo en las economías respectivas. El estudio se acaba de publicar y se basa en datos de 2013 :

- Gibraltar representa 1 de cada 4 puestos de trabajo en la región del Campo de Gibraltar y Gibraltar

- En el 2013 la economía de Gibraltar (PIB £847m) representó una cuarta parte del PIB del Campo de Gibraltar.

- Empresas de Gibraltar compraron $£ 381 \mathrm{~m}$ ( $€ 533 \mathrm{~m}$ ) de mercancias y servicios procedentes de España en 2013.

- Los trabajadores transfronterizos ganaron $£ 126 \mathrm{~m}(€ 145 \mathrm{~m})$ en sus puestos de trabajo en Gibraltar, la mayoría de los cuales de España, por lo tanto, generaron más crecimiento económico en España.

\section{Gibraltar}

El Reino Unido se incorporó a la Comunidad Económica Europea (CEE) en enero de 1973. Asimismo Gibraltar como Territorio Británico de Ultramar se convirtió en miembro de la CEE al mismo tiempo. España se incorpora como miembro en 1986, reconociendo en su condición de país integrante de la Unión Europea, la apertura de la frontera con Gibraltar y garantizar el libre acceso y circulación, por lo que cumple con el Artículo 3 (c) del Tratado de Roma. La frontera para peatones había sido abierta en diciembre de 1982 tras un período de 13 años de cierre. A los vehículos (automóviles, autobuses y camiones) no se les permitan el libre acceso para cruzar la frontera con España hasta febrero de 1985.

La población gibraltareña alcanzó el número de 33.000 aproximadamente de habitantes en el año 2014.

REGLAMENTO DE FUNCIONAMIENTO GRUPO TRANSFRONTERIZO 
La frontera entre Gibraltar y España ha sido una fuente de fricción y tensión desde entonces. Se han hecho varios intentos de resolver las cuestiones pendientes entre los gobiernos de Gibraltar, el Reino Unido y España en el transcurso de los años. Si bien se ha logrado algún éxito en la solución de estas cuestiones, pero no tienden a durar mucho tiempo.

Los intentos más recientes por abordar el problema del flujo fronterizo formaron parte de un comunicado conjunto anunciado en Córdoba el 18 de septiembre de 2006 por los gobiernos del Reino Unido, del Reino de España y Gibraltar.

Esta situación de buena vecindad, y de alcanzar acuerdos específicos para los problemas concretos, cambian en la última etapa, alcanzando una situación extremadamente dañina para los ciudadanos en Julio de 2013 , con la puesta en marcha de los estrictos controles en la frontera, sin recursos suficientes.

Estos hechos son los que animan a los agentes sociales y económicos a promover un grupo permanente con el objetivo de fortalecer las relaciones, promover sinergias y apostar por la cooperación como mecanismo de defensa de los intereses de los ciudadanos y ciudadanas de Gibraltar y La Comarca

\section{OBJETIVO GENERAL DEL GRUPO}

Este grupo se constituye el 7 de enero de 2014, con vocación permanente y con el fin de defender los intereses comunes de la ciudadanía de ambos lados, desde el firme convencimiento de que el dialogo y la cooperación son inexcusables para el desarrollo social y económico de ambas sociedades, siendo elementos consustanciales a las relaciones entre dos territorios que forman parte de la Unión Europea.

REGLAMENTO DE FUNCIONAMIENTO GRUPO TRANSFRONTERIZO 
V. Grupo Transfronterizo/Cross-Frontier Group, Memorándum de Entendimiento con el Gobierno de Gibraltar (2017) y

Reglamento de funcionamiento (2018)

\section{DOC}

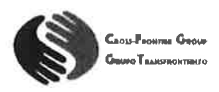

Las partes constituyentes del grupo como establecido en el 7 enero de 2014 se considerarán como miembros permanentes del grupo, además de las organizaciones empresariales integradas en el año 2017 (Confederación de Empresarios de Cádiz y Cámara de Comercio del Campo de Gibraltar).

La inclusión de otras fuerzas al grupo será por voto unánime de los miembros permanentes.

\section{FUNCIONES}

Atendiendo al objetivo general podemos concretar las siguientes funciones generales, cuyas actuaciones concretas se planificaran de forma consensuada entre los integrantes del Grupo.

1.- Promover actuaciones de cooperación para el desarrollo social y económico de la zona

2.- Formular propuestas a las administraciones y otras instituciones de carácter público o privado para el impulso del desarrollo económico y social endógeno a partir de las potencialidades y oportunidades que ofrece el territorio.

3.-Reforzar vínculos transfronterizos valorizando la complementariedad de las actividades económicas, de la innovación y del capital humano.

4.- Mejorar la calidad de vida de la población a través de estrategias comunes.

\section{$\underline{\text { Objetivos estratégicos }}$}

EI GTF nace a raiz de los problemas fronterizos, por lo tanto no hay que olvidar que el propósito inicial fue el del resaltar los problemas tanto de ámbito socioeconómico como el de libre movimiento de personas y mercancias.

REGLAMENTO DE FUNCIONAMIENTO GRUPO TRANSFRONTERIZO 
Dentro de este marco de colaboración y cooperación se consideran prioritaria para el desarrollo de las relaciones transfronterizas y el cumplimiento del objetivo general:

-Competitividad, innovación, empleo y formación;

-Turismo y cultura;

-Accesibilidad, logística.

-Internacionalización y cooperación transfronteriza.

- Otros.

A un nivel más operacional, podemos definir Objetivos Estratégicos, orientados hacia la actuación.

1.- Proponer actuaciones orientadas a desarrollar un espacio económico transfronterizo competitivo e innovador

Mediante el impulso de redes de servicios de apoyo a las empresas, en una lógica transfronteriza., el desarrollo y utilización de tecnologías de la información y comunicación a escala transfronteriza., fomento de la investigación, promover recursos transfronterizos como apoyo al desarrollo sostenible, poner en valor el patrimonio histórico y cultural, generar la protección y utilización eficiente de los recursos.

2.- Estimular el vínculo transfronterizo mediante el impulso del empleo apostando por una formación profesional de calidad. Propiciando una mejora tanto en la interrelación del mercado laboral como social de la población transfronteriza.

3.- Valorizar los territorios como instrumento de desarrollo y de cohesión, mediante la mejora de la accesibilidad, la conectividad, y la movilidad de las personas $y$ de bienes tangibles e intangibles.

REGLAMENTO DE FUNCIONAMIENTO GRUPO TRANSFRONTERIZO 
V. Grupo Transfronterizo/Cross-Frontier Group, Memorándum de Entendimiento con el Gobierno de Gibraltar (2017) y

Reglamento de funcionamiento (2018)

\section{DOC}

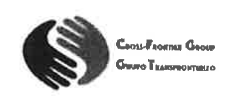

\section{COMUNICACIÓN}

Una buena comunicación interna es indispensable para alcanzar los objetivos comunes En esta óptica, deben introducirse diversas herramientas con el fin de optimizar los flujos de comunicación interna, entre las cuales se deben destacar:

- Reuniones periódicas de coordinación (min 4 anuales)

- Mailing a los miembros del GTF sobre cualquier tipo de acción de comunicación.

-- Whats App

-- Página web

\section{La comunicación externa:}

Las acciones de comunicación pueden dirigirse a tres tipos de público:

- Medios de comunicación.- Informar cuando el grupo considere oportuno con notas de prensa a los medios de comunicación, profesionales del periodismo y responsables de comunicación en instituciones y administraciones públicas.

- Crear y organizar acontecimientos de interés local y regional susceptibles de estar bien cubiertos por los diferentes medios de comunicación

- Instituciones públicas, partidos políticos, administraciones, etc, desarrollar actuaciones de comunicación con el fin de informar y realizar propuestas de las acciones.

REGLAMENTO DE FUNCIONAMIENTO GRUPO TRANSFRONTERIZO 
Identidad corporativa:

EI logo del GRUPO, para su uso en todas las actividades de comunicación interna y externa realizadas, es:

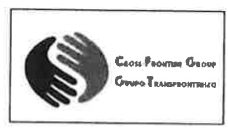

Este logo podrá ser modificado por acuerdo de los componentes del grupo.

\section{ORGANOS}

\section{COMISION PERMANENTE EJECUTIVA}

Estará integrado por 2 miembros permanentes de las organizaciones integrantes, nombrados por las propias organizaciones.

Los miembros serán nombrados y acreditados ante la secretaria de la GTF por sus respectivas organizaciones, reservándose las representadas el derecho a su cese y sustitución.

Las funciones a desarrollar por los miembros:

- Asistir a las sesiones y participar en los trabajos a desarrollar

- Tener disponibilidad para asistencia a actos y eventos convocados por el Grupo o a instancia del mismo.

- Acceder a la documentación, información, actas, etc que obre en Secretaria.

- Aprobación de resoluciones y acuerdos

REGLAMENTO DE FUNCIONAMIENTO GRUPO TRANSFRONTERIZO 
V. Grupo Transfronterizo/Cross-Frontier Group, Memorándum de Entendimiento con el Gobierno de Gibraltar (2017) y

Reglamento de funcionamiento (2018)

\section{DOC}

\section{PRESIDENCIA Y SECRETARIA}

\section{De la Presidencia y Vicepresidencia:}

La designación de la presidencia y vicepresidencia deberá recaer en dos de sus miembros y se elegirá, de forma alternativa, entre la representación empresarial y sindical y por periodo de un año teniendo en cuenta la nacionalidad, siendo esta alterna idealmente, de acuerdo con el orden de prelación que se consensúe entre los miembros.

Una vez finalice el periodo de mandato, el vicepresidente pasaria a ser Presidente automáticamente y los miembros del grupo tendrían que elegir la vacante de vicepresidencia a la organización que por turno corresponda. Funciones presidencia:

- Convocar, presidir y moderar las sesiones

- Formular el orden del día de las sesiones, debiendo incluir los puntos que soliciten cualquiera de sus miembros preferentemente con 7 días de antelación.

- Dirigir las deliberaciones y mantener el orden de las reuniones.

- Realizar la portavocía ante los medios de comunicación mediante una óptima coordinación entre los portavoces (Presidente y Vicepresidente) de los 2 lados de la frontera.

- Y cualesquiera otras que les sean encomendadas.

\section{De la Secretaría y Vicesecretaria:}

La Secretaría y Vicesecretaria será ejercida por dos miembros asignados de forma rotativa cada año. Sus funciones serán asistir a las reuniones y levantar acta de lo acordado. Asimismo certificaran el

\section{REGLAMENTO DE FUNCIONAMIENTO GRUPO TRANSFRONTERIZO}


contenido de las actas, y tendrán la custodia de la documentación recepcionada, dando curso a los acuerdos que se adopten, y la realización de cuantas actuaciones sean precisas para el correcto funcionamiento.

Se encomienda a la secretaría realizar y enviar notas de prensa que se aprueben a los medios de comunicación; así como cuantas otras que se deleguen por el GTF.

\section{AREAS ESTRATEGICAS}

Con el fin de dar respuesta concreta a los objetivos estratégicos, el grupo podrá constituir a propuesta de al menos dos de las entidades miembro, siempre por unanimidad con carácter permanente o para cuestiones específicas, todas aquellas áreas para el análisis, desarrollo de propuestas, instrumentos de ejecución y para cuantas actuaciones operativas sean imprescindibles para la consecución de forma eficaz de los objetivos manifiestos.

Las resoluciones o acuerdos de estas deberán ser ratificados unánimemente por Comisión Permanente Ejecutiva.

En la composición de las áreas se deberá respetar la proporcionalidad y la presencia de todas las organizaciones representadas.

La representación de las organizaciones pertenecientes a las áreas será flexible, pudiendo estar integrada por más miembros, técnicos, asesores, etc...así como por personal experto ajeno al GTF que los miembros consideren de importancia relevante su aportación al tema a tratar. Toda persona ajena al grupo podrá participar pendiente previa consulta del grupo y por acuerdo unánime.

REGLAMENTO DE FUNCIONAMIENTO GRUPO TRANSFRONTERIZO 
V. Grupo Transfronterizo/Cross-Frontier Group, Memorándum de Entendimiento con el Gobierno de Gibraltar (2017) y Reglamento de funcionamiento (2018)

\section{DOC}

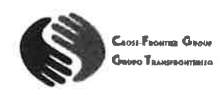

SEDE

Donde este la secretaria estará la sede durante el tiempo que dure el cargo.

\section{MODIFICACIONES DEL PRESENTE DOCUMENTO}

El presente documento podrá sufrir cuantas modificaciones se consideren necesarias para un mejor funcionamiento y consecución de los fines previstos. Siempre a propuesta de, al menos, dos de las organizaciones miembros de GTF y tras respaldo unánime de sus componentes.

26 de enero de 2018
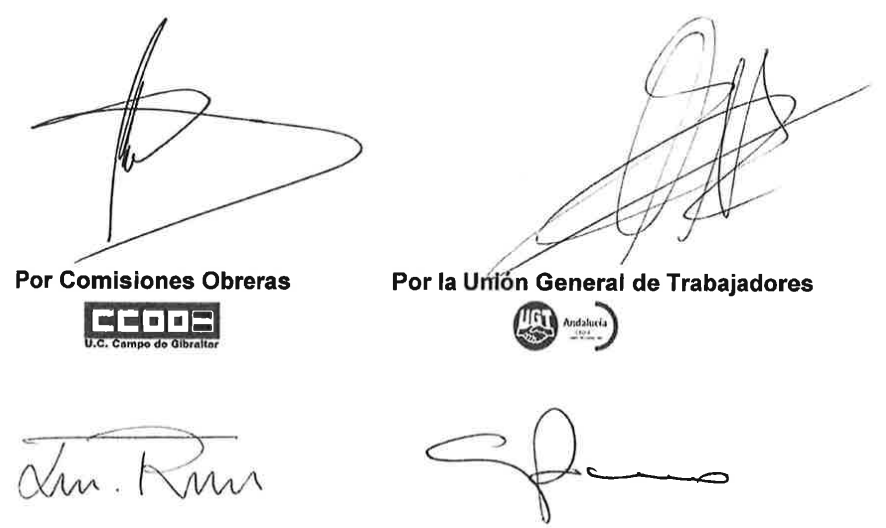

Por APYMELL

Por Confederación Empresarios de Cádiz

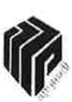




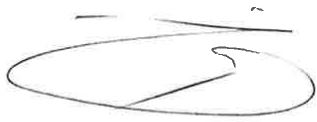

Por Cámara Comercio C.G.

Cámmal’a

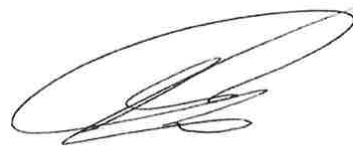

Por Cámara Comercio Gib.

(x)

(5)

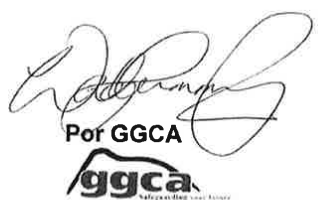

Por Unite The Unión

unite

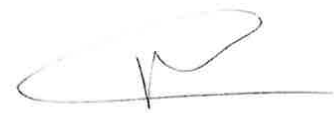

Por GTA / NASUWT

NASUYT

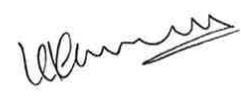

Por GFSB

GFSB 
V. Grupo Transfronterizo/Cross-Frontier Group, Memorándum de Entendimiento con el Gobierno de Gibraltar (2017) y

Reglamento de funcionamiento (2018)
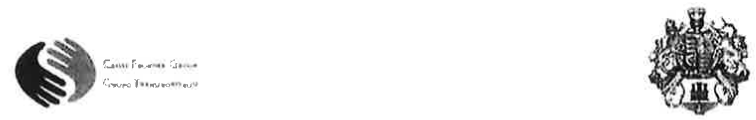

MEMORANDUM DE ENTENDIMIENTO PARA EL FORTALECIMIENTO DE LA COOPERACION ENTRE EL GOBIERNO DE GIBRALTAR Y EL GRUPO TRANSFRONTERIZO DE GIBRALTAR Y EL CAMPO DE GIBRALTAR.

(1) Este memorándum de entendimiento se introduce en 7 de junio de 2017 entre el gobiemo de Gibraltar y cl Grupo Transfronterizo con la intención de ambas partes de facilitar y promover el crecimiento en las economías del Campo de Gibraltar y Gibraltar. El Gobierno de Gibraltar y el grupo transfronterizo, inediante el presente memorándum, transmiten las ventajas que ofrece Gibraltar para el Campo de Gibraltar y viceversa. El impacto de la Economía en positivo entre ambas zonas podría ser todavía mayore y generar mucho más riqueza.

(2) El presente documento de entendimiento tiene como objetivo establecer un compromiso de cooperación y colaboración para mantener una frontera fluida y desarrollar el potencial económico así como mejorar las oportunidades de trabajo a ambos lados de la frontera.

(3) Este memorándum compromete a ambos firmantes a trabajar juntos y compartir recursos mientras estos sean de beneficio para sus organizaciones, y para el público en general de ambos lados de la frontera.

(4) MODALIDADES DE COOPERACION

Las partes, podrán promover la colaboración a la cual se hace referencia en la introducción del memorándum bajo las siguientes modalidades en los distintos ámbitos y de la gestión de ambas partes.

Intercambio de información.

Desarrollo de proyectos conjuntos $y$ actividades de apoyo a los mismos.

Intercambio de especialistas y recursos humanos y materiales.

Realización de talleres, conferencias y seminarios en las áreas de cooperación.

Uso de instalaciones y recursos educativos para formación.

Trabajar en mantener y mejorar la fluidez en el tránsito de personas.

Trabajar en mantener y mejorar la fluidez de vehículos y mercancías, creando un marco legal para tal fin.

Mantenimiento y creación de nuevos empleos.

Incrementar la demanda de bienes y servicios en los distintos sectores productivos

Ofrecer facilidades, información y ayudas en la gestión a las empresas de Gibraltar y el Campo de Gibraltar que se quieran establecer a un lado y otro de la frontera.

Impulsar la creación de una zona fiscal en la Línea de la Concepción para que las empresas Gibraltareñas puedan abrir delegaciones, como fuente de creación de empleo para todo el Campo de Gibraltar y Gibraltar.

Investigar modos alternativos del desarrollo comercial y cooperación a ambos lados de la frontera. 
(5) Estará a cargo una Subcomité acordado por ambas partes para coordinar actividades especificas entre los firmantes de este memorándum de entendimiento, acordado entre ambas partes donde miembros contribuyendo a tal, podrian tener el apoyo de agencías externas este MDE.

(6) Todos los firmantes están obligados a tratar con estricta confidencialidad todos los asuntos discutidos y sólo se informará a los medios de comunicación de los acuerdos tangibles o áreas de cooperación, bajo acuerdo de todos los firmantes.

(7) El presente Memorándum de Entendimiento entrará en vigor a partir de la fecha de su firma y continuará vigente por un periodo de tres años, prorrogable automáticamente por periodos iguales, a menos que cualquiera de las partes manifieste su decisión de darlo por terminado, mediante comunicación escrita.

(8) El presente Memorándum podrá ser modificado por mutuo consentimiento de las partes formalizando a través de comunicaciones escritas, en las que se especifique la fecha de su entrada en vigor.

(9) Este memorándum está libre de acuerdos independientes celebrados entre cualquiera de los firmantes, ya sea entre los miembros del Grupo Transfronterizo como el gobierno de Gibraltar y también está libre de afiliaciones políticas independientes o colcctivas.

(10) La terminación anticipada del presente documento no afectará la conclusión de las actividades de cooperación que hubieran sido formalizadas durante su vigencia.

Firmado en Gibraltar el día 7 de junio de 2017 


\section{DOCUMENTACIÓN}

V. Grupo Transfronterizo/Cross-Frontier Group, Memorándum de Entendimiento con el Gobierno de Gibraltar (2017) y Reglamento de funcionamiento (2018)

Firmado en Gibraltar el día 7 de junio de 2017

HM GOVERNMENT OF GIBRALTAR:

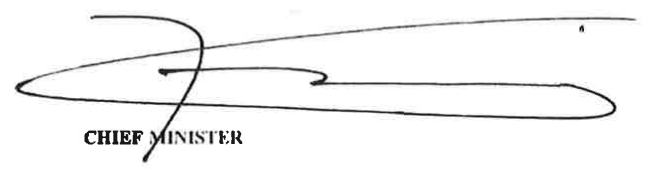

POR EL GRUPO TRANSFRONTERIZO:

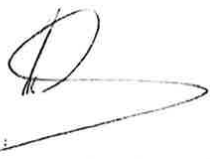

COMISIONES OBRERAS (PRESIDENCIA)
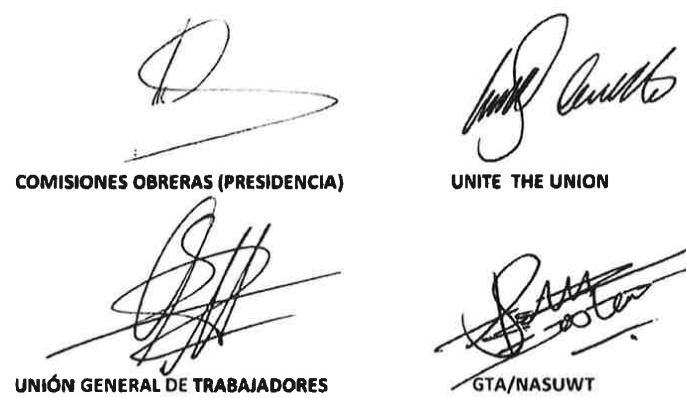

UNITE THE UNION

UNIÓN GENERAL DE TRABAJADORES
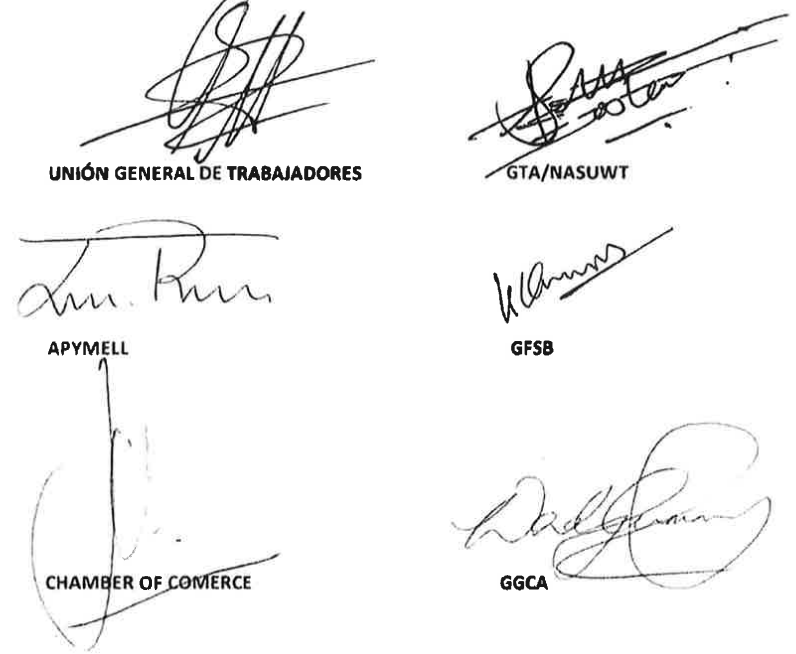

GFSB

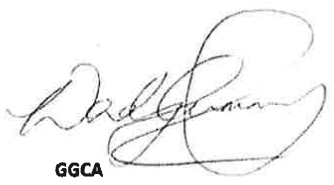




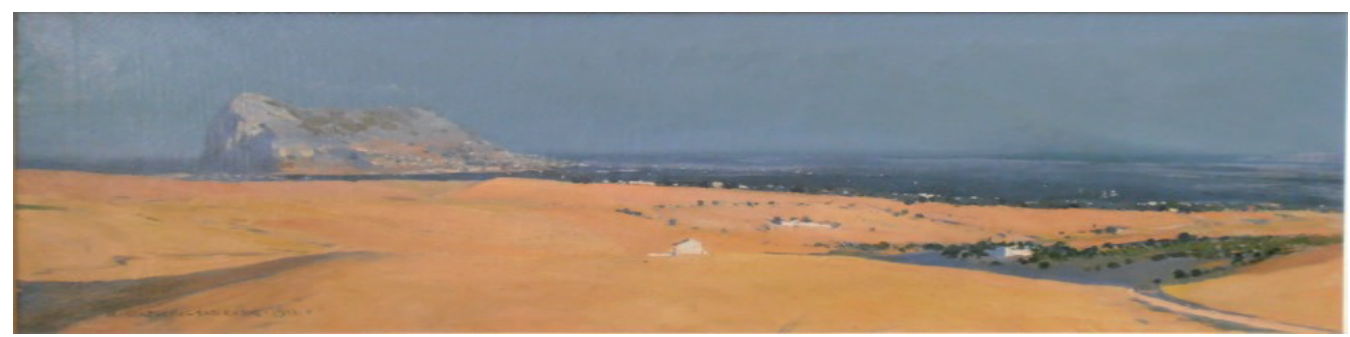

\section{Cuadernos de Gibraltar Gibraltar Reports}

\section{\#04 | 2020-2021}

Sumario

Table of Contents

\section{EDITORIAL}

Alejandro DEL VALLE GÁLVEZ; Inmaculada GONZÁLEZ GARCÍA, Gibraltar after the Brexit: Looking for a New and Imaginative Model of Cross-Border Cooperation in the EU Framework

\section{ESTUDIOS Y NOTAS}

Luis M. HINOJOSA MARTÍNEZ, Los movimientos de capital y los servicios financieros en las relaciones con Reino Unido y Gibraltar tras el Brexit: ¿Seguridad jurídica o ley de la selva?

Luis ROMERO BARTUMEUS, Las escalas de submarinos nucleares en Gibraltar y Rota, y los Planes de Emergencia Radiológica

Facundo D. RODRÍGUEZ, La cuestión de las Malvinas (Falklands) en el Cuarto Decenio Internacional para la Eliminación del Colonialismo

\section{ÁGORA}

Alejandro DEL VALLE GÁLVEZ, Creación de una AECT-Agrupación Europea de Cooperación Territorial- Campo de Gibraltar/Gibraltar (Guía breve sobre planteamiento, requisitos y procedimiento)

Alejandro GARCÍA HEREDIA, El Acuerdo Fiscal entre España y el Reino Unido en relación con Gibraltar

David CHEVASCO, Notes on Contemporary Bilingualism, Llanito and Language Policy in Gibraltar: a Study with a Present-day View of Linguistic Challenges

\section{DOCUMENTACIÓN}

Inmaculada GONZÁLEZ GARCÍA; Alejandro DEL VALLE GÁLVEZ; Lorena CALVO MARISCAL, Juan D. TORREJÓN RODRÍGUEZ:

Documentación I. Naciones Unidas - Gibraltar - Decisiones aprobadas por la Asamblea General de la ONU (2017-2019)

Documentación II. Brexit y Gibraltar - Tratado de Retirada, Protocolo, Tratado Fiscal y MOUs (2018-2020)

Documentación III. España, Brexit y Gibraltar. Declaraciones, Comunicados y Notas de Prensa (2018-2020)

Documentación IV. Andalucía, Brexit y Gibraltar (2018-2019)

Documentación V. Grupo Transfronterizo/Cross-Frontier Group, Memorándum de Entendimiento con el Gobierno de Gibraltar (2017) y Reglamento de Funcionamiento (2018)

Documentación VI. Principio de Acuerdo entre España y el Reino Unido sobre Gibraltar y sobre el Brexit, de 31.12.2020

Documentación VII. Mandato negociador para el Tratado entre el Reino Unido y la Unión Europea con respecto a Gibraltar, 5 de octubre de 2021

\section{LOS ARCHIVOS DE LUIS ROMERO BARTUMEUS}

La descolonización de Gibraltar, de CALVAR, J. - GUERITZ , E. J. - DEL CAMPO, S. - DAVIS H., Ed. Instituto de Cuestiones Internacionales, 1981, 110 páginas, por Luis ROMERO BARTUMEUS

Matar al Mensajero, Vivencias de un «Palomo» en Gibraltar, de TRIAY BOZZINO, J. J. - CASAUS BALAO, J. A., Ed. : Colecciones AUREA, 2000, 198 páginas, por Luis ROMERO BARTUMEUS

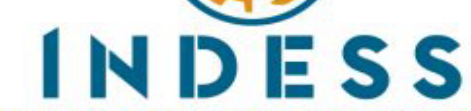

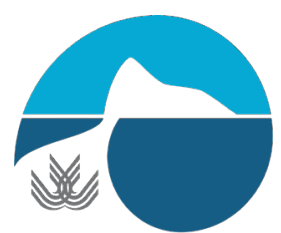

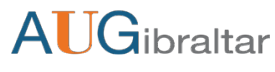

CÁTEDRA JEAN MONNET INMIGRACIÓN Y FRONTERAS DERECHO DE LA UNIÓN EUROPEA $\sim 2$

EDUCACIÓN DE LA UNIÓN EUROPEA

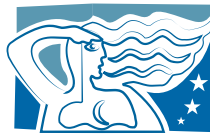

ESTUDIOS

INTERNACIONALES Y EUROPEOS

Centro de Estudios Internacionales y Europeos del Área del Estrecho 